\title{
Review of Metal AM Simulation Validation Techniques
}

\author{
Aaron Flood and Frank Liou \\ Department of Mechanical and Aerospace Engineering, Missouri University of Science and Technology, Rolla 65409, USA
}

\begin{abstract}
Due to the complexity of metal AM (additive manufacturing), it can require many trial runs to obtain processing parameters which produce a quality build. Because of this trial and error process, the drive for simulations of AM has grown significantly. A simulation only becomes useful to researchers if it can be shown that it is a true representation of the physical process being simulated. Each process being simulated has a different method of validation to show it is an accurate representation of the process. This paper explores the various methodologies for validation of laser-based metal AM simulations, focusing mainly on the modeling of the thermal processes and other characteristics derived from the thermal history. It will identify and explain the various validation techniques used, specifically looking at the frequency of reported use of each technique.
\end{abstract}

Key words: AM simulation, simulation validation, heat transfer modeling, stress modeling, micro-structure modeling.

\section{Introduction}

AM (additive manufacturing) is a complex process and many have attempted to generalize the process using mathematical models. In order to show the validity of each model, researchers have developed methods to compare the results from these simulations to experiments which can be performed. Each aspect of the AM process which is being simulated will have a different technique for validation. The main phenomena of AM which have been studied are heat transfer, induced stress, and microstructure. For each of these phenomena, the various validation techniques which have been used in literature will be investigated including a brief description of the technique fundamentals.

There are two main methods of validation for the modeling of the thermal history, instrumental and indirect. The instrumental methods utilize a hardware setup to directly measure the temperature of the process at a specific location. Whereas the indirect methods compare a different physical characteristic, such as melt pool depth, which is linked to the temperature, this is then used to validate the temperature profile.

Corresponding author: Aaron Flood, PhD candidate, research field: metal additive manufacturing simulation.
To validate the stresses which are induced on the part, qualitative and quantitative approaches have been utilized. To qualitatively validate the results, some have looked for the generation of cracks and compared these results to a simulation. This validation can give a gross comparison of the simulation and experiments. A simple method of gathering a quantitative comparison is to measure the distortion of the final part. This can either be done using a laser displacement sensor, in situ, or a 3-D scanner after the deposition is complete. These results, though more precise than crack generation, are not extremely precise. To precisely measure the strain, it is necessary to gather a diffraction pattern, either with $\mathrm{X}$-rays or neutrons, for the part. This allows for the precise locations of the atoms to be known which gives the exact values for the strain in the part.

Due to the drive for AM from the aerospace industry, many researchers are focusing on Ti-64 (Ti-6Al-4V) as their material of choice. Therefore that will be the focus of the microstructure section of this paper. Even though the focus is Ti-64, all the methods which are presented can be generalized to any metal. The first method of comparison is to compare the phase which occurs, usually on a pixel by pixel basis or a voxel by voxel basis for 3-D. This will give a general comparison and limited quantitative comparison of the experiment and simulation. If a more detailed comparison is desired, 
then in addition to the phase the grain sizes can be compared. This comparison is usually made by comparing the size distribution of the phases.

\section{Heat Transfer Validation Techniques}

The most fundamental, and first developed, process in AM which has been modeled is the flow of heat through the part. This problem was first tackled by researchers focusing on simulating the welding process, and much can be derived from their work. A very extensive review was done by Mackwood and Crafer [1] from which key elements can be utilized. The first numerical solutions which can be applied to the problem of AM, by Mazumder and Steen [2], created a 3-D finite difference model to simulate a Gaussian laser on a semi-infinite workpiece. Their model did not include temperature dependent material properties, which was later remedied by Chande and Mazumder [3]. This later iteration also accounted for latent heat of phase change which has recently proven to be an important aspect of AM simulations. The last simulations developed, which are the most applicable to $\mathrm{AM}$, are for multi-pass welding by Reed and Bhadeshia [4], Lindgreen et al. [5], and Frewin and Scott [6]. In these models, the laser is passed over the same area multiple time to determine the heat flow due to the multiple passes. These simulations were the first time that "quiet" elements were utilized. These elements are considered inactive until the part has been built up to their location. At that time, they are activated and are included in the simulation. This model has been the foundation that most AM simulations have been built upon.

In order to validate these models, thus far in the literature, there have been two approaches. The first is to validate the thermal model with an instrument equipped to measure temperature. If this has not been done, then the researchers will measure another physical characteristic of the build and use that to show the model's validity. A representative set of papers have been presented in Table 1.
Table 1 Breakdown of validation techniques.

\begin{tabular}{lll}
\hline \multicolumn{2}{l}{ Instrument validated } & Physical char. validated \\
\hline IR/CCD camera & {$[7-10]$} & Melt pool depth [11-13] \\
Pyrometer & {$[14,15]$} & \\
Thermal couple & {$[15-17]$} & \\
\hline
\end{tabular}

These papers show that more attempts have been made to validate the models using instrumental validation as opposed to using another physical characteristic. This is most likely due to the direct link between the measured value and the simulated value. When using another physical characteristic, it is necessary to know the exact linkage between the trait being measured and the one being simulated. For this reason, there are more opportunities for error and false validation, or rejection, of a given model. From the literature reviewed, there are three prominent instruments which have been used to validate the models.

The most common instrument used is an IR or CCD camera, these cameras are appealing based on several features. The first key feature is that this is a non-contact measurement, this means that it is applicable to every form of metal AM to date. Cameras are also capable of capturing data at a high frame rate, Hu and Kovacevic [7] report frame rates as high as 800 frames/sec. Coupled with this frame rate is the camera's resolution, which Kolossov et al. [8] report using a camera of $256 \times 256$ pixels where each pixel is $0.1 \times 0.1 \mathrm{~mm}$. A final key feature is its ability to be used in-situ, which can allow for it to be used as feedback control if a closed loop system is used. These capabilities allow researchers to quickly and accurately assess the surface temperature of a build. This method of measuring temperature is not without its faults. The first, according to Wegner and Witt [18], is that these cameras are very sensitive to the angle and the distance they are placed from the object begin measured. Additionally, according to Fischer et al. [19], these cameras measure the average temperature of the skin of the object during the time elapsed for 1 frame. This problem does not apply to CW lasers, however, when 
using a pulsed laser, the skin temperature can spike very rapidly which can result in inaccurate measurements.

$$
\begin{gathered}
Q_{r}=\int I(\lambda, T) \delta \lambda d A \\
T_{e f f}=\frac{h c}{\lambda \sigma \ln \left(1+n / \sum_{i=1}^{n}\left(e^{\frac{h c}{\lambda \sigma T_{i}}}-1\right)^{-1}\right)}
\end{gathered}
$$

The next instrument most commonly used is a pyrometer, which is a non-contact spot measurement which can be used in-situ. This results in the ability to measure the average temperature of a specific area. This is not as useful as cameras previously presented due to the lack of resolution. However, because of their simplicity, it is possible to create a mathematical model to predict the pyrometer output. This can be done by knowing the power of the thermal radiation which returns to the pyrometers and is shown in Eq. (1) [20], where $\mathrm{I}(\lambda, \mathrm{T})$, is the spectral distribution of the blackbody emissive power given Planck's radiation law. It is possible to then integrate Eq. (1), assuming that the laser is a Gaussian heat source and that the pyrometer is sampling a $1 \mathrm{~mm}$ radius, it is possible to solve for the effective temperature that the pyrometer reads, Eq. (2), where $h$ is Planck's constant, $c$ is the speed of light, $\lambda$ is the wavelength of the emitted radiation, $\sigma$ is Stefan-Boltzmann constant, $n$ is the number of small sampling areas within the pyrometer viewing area, and $T_{i}$ is surface temperature within the small $n$ areas.

This has allowed for Dia et al. [14] to create a simulation which includes a pyrometer to control the laser power. This simulation can predict the changes that the pyrometer will make to the laser power, for a closed loop system, to keep a constant melt pool size.

The last method found in the literature to measure the temperature directly utilizes thermocouples, which are contact spot measurements. The fact that they must be fixed, welded in most cases, to the surface makes them impractical for some applications, such as powder bed process. In addition, they will only record the average temperature of a specific location. Therefore, to obtain an accurate representation of the temperature profile, several thermocouples need to be placed on the working surface. Another downfall with thermocouples is their inability to measure the melt pool temperature. Since they need to be fixed to the surface, if an attempt is made to measure the melt pool they will become detached from the substrate and the data will be invalid. For these reasons, current researchers have only used thermocouples as a secondary validation technique and utilize another technique for the main source of data.

Besides these direct methods of validating the thermal modeling, some researchers have taken the approach of measuring a more easily attained data set and compared that to the simulation, namely the melt pool size and the shape of the build. In this method a simple surface laser heating simulation and experiment are performed, where the laser is simply used to melt a track on the surface of the substrate. In the experiments, a slice is taken perpendicular to the laser path which is then analyzed, typically with an optical microscope. This allows for the width and depth of the melted region to be measured, as seen on the left image in Fig. 1. In the simulation, since the temperature is tracked for each element, it is possible to flag elements which have melted, this is done in the right image in Fig. 1 by changing their color to red. In addition to the use of the surface laser heating, some have simulated a single track build, which can be seen in Fig. 2 .

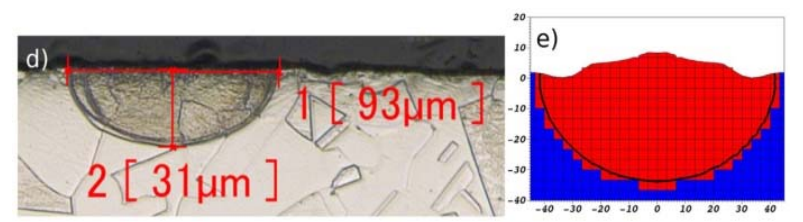

Fig. 1 Validation of thermal analysis by comparing melt pool dimensions of experiment (left) and simulation (right) [12].

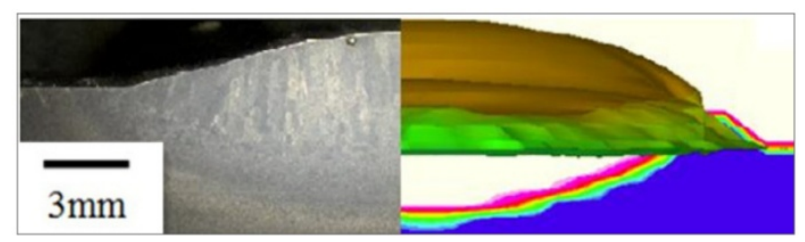

Fig. 2 Validation of thermal analysis by comparing single track build dimensions of experiment (left) and simulation (right) [13]. 
Table 2 Applicability of validation techniques to basic AM processes.

\begin{tabular}{lll}
\hline & Powder bed & DED \\
\hline IR/CCD camera & $\mathrm{X}$ & $\mathrm{X}$ \\
Pyrometer & $\mathrm{X}$ & $\mathrm{X}$ \\
Thermal couple & & $\mathrm{X}$ \\
Melt pool depth & $\mathrm{X}$ & $\mathrm{X}$ \\
\hline
\end{tabular}

Table 3 Highest accuracy reported of validation techniques to basic AM processes*.

\begin{tabular}{lll}
\hline & Response Time & Resolution \\
\hline IR/CCD camera & $800 \mathrm{fps}$ [7] & $10.9 \mathrm{um}^{2}[8]$ \\
Pyrometer & & $3 \mathrm{~mm}^{2}[14]$ \\
Thermal couple & & $0.2 \mathrm{~mm}^{2}[15]$ \\
\hline
\end{tabular}

*Values not reported are left blank.

This indirect method of validation can typically be done without specialty equipment. However, this method of validation introduces new complications which can hide, or skew, the results. Since the material is melted, the flow of the molten material dictates the shape of the melt pool. For that reason, this validation technique requires that both the thermal and fluid models are correct. Therefore, the direct methods are simpler to implement than the indirect methods.

In general, these methods all have different applicability to the various metal AM processes. As can be seen in Table 2, all the methods of validation are applicable to DED (directed energy deposition) metal AM. When working with a powder bed process, on the contrary, it is impossible to use a thermocouple as previously stated. For this method of metal AM, it is necessary to use one of the non-contact methods. When looking at the accuracy of the methods, displayed in Table 3, the camera system will usually have the highest resolution and response time, but will also be the most expensive. Therefore, it is necessary to balance the cost and the accuracy needed.

\section{Stress Validation Techniques}

Inherent in AM processes, is a cyclic heating which leads to stresses being induced. The stressing process has been divided into four stages by Ding et al. [21].

Stage A occurs when the heat source approaches the location of interest on the part. This stress is compressive since the volume under the heat source is expanding. This compressive stress is elastically compensated for by the material until the compressive yield stress limit is surpassed. When the compressive yield limit is surpassed, stage B takes place. In this stage, plastic flow of material occurs and the compressive stress is reduced. Stage $\mathrm{C}$ has begun when the material begins to cool which results in tensile stress. These stresses are caused by the contraction of the surrounding material. They remain elastic until the tensile yield stress is surpassed. The final stage of stress is stage $\mathrm{D}$, which occurs when the tensile yield limit is surpassed and plastic flow begins. These stresses can all be derived from the thermal history of a specific location and its neighbors. Due to the difficulty of measuring the stress, only a few methods have been used throughout literature as displayed in Table 4.

One of the simplest, though not accurate method, is to observe the creation of cracks within the part and compare that to simulation results. This method, used by Zhu et al. [9], is simple and can be done without any specialty equipment. This method, however, due to its lack of precision, can only be used to qualitatively verify that a simulation is giving results which generally agree with the experiment. This method cannot be used to quantitatively validate a mathematical model.

If a more refined approach is needed Liu et al. [22] have looked at build plate deformation as a link between the simulation and the experiment. Thus far in the literature, this has been implemented by using a laser displacement sensor or a 3-D scanner to measure the distortion which occurs in the final part. To use a laser displacement sensor, as shown in Fig. 3, one edge

Table 4 Frequency of stress analysis techniques.

\begin{tabular}{ll}
\hline Presence of cracks & {$[9,23]$} \\
Final part distortion & {$[17,22,24]$} \\
DIC (digital image correlation) & {$[25,26]$} \\
Neutron diffraction & {$[16,21]$} \\
X-ray diffraction & {$[27,28]$} \\
\hline
\end{tabular}




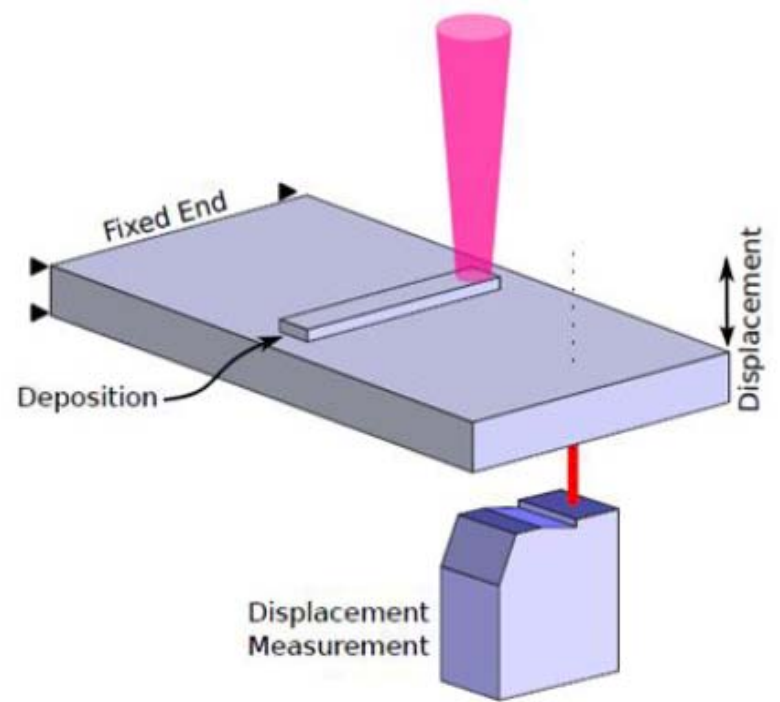

Fig. 3 Experimental setup using laser displacement sensor to measure distortion [23].

of the build plate is clamped creating a cantilever, and the sensor is used to monitor the free end. This edge of the build plate is monitored in real time to determine the fluctuations that occur during the build. These fluctuations are then correlated to the distortions which are seen in the simulation. When done correctly, the stress which occurs in the part can be correlated to the simulation to show the accuracy of the simulation. This method, in addition to measuring the stresses as they occur, has the added capability to measure the residual stresses which build up throughout the entire process. One problem with this setup is that the depositions location on the substrate is critical for accurate results. This is simple in the simulation, however, in the experimental setup, this can prove challenging. The other method of measuring the induced stresses is to build the part and upon removal from the machine, to use a 3-D scanner to measure distortions. This will give the final dimensions of the part and a more complete picture can be gained using this approach.

Each of these approaches has its advantages. If a full picture of the part is needed, then a 3-D scanner should be utilized. This is because the scanner inspects the whole part, or at least a larger section of the part, compared to the laser displacement sensor which only monitors a single point.
However, if more accurate results are needed, then a laser displacement sensor should be used. The laser sensor used by Heigel et al. [17] reports an accuracy of $\pm 1 \mu \mathrm{m}$, whereas the 3-D scanner used by Denlinger et al. [24] reported an accuracy of $\pm 500 \mu \mathrm{m}$.

Another method of obtaining the distortion, or the surface stresses induced, of the part is DIC. The process of DIC uses a camera to observe the part and sense any motion which is induced on the part. Pan et al. [29] describe how this method tracks points which are placed on the part to determine their relative motion to calculate the stresses and distortion a part endures. An example of how the points move can be seen in Fig. 4. This method will inherently give the distortion of the part. However, Wu et al. [25] showed that it is possible to precisely determine the surface level stresses which are induced on the part. This is done by selectively stress relieving the part through sectioning, hole drilling, or slitting. These methods allow for the distortion that occurs to be related back to the stress which the part is experiencing. The main drawback to this method of validation is that it is a destructive method. However, one of the main advantages of this method is that the resolution is limited by the camera which is being used. The motion of the material is measured in pixels on the camera. That results in the ability to have a fine resolution if a high-resolution camera is used. The resolution of the camera can also be supplemented by attaching the camera to a microscope. This technique can greatly increase the detail which can be observed with the DIC method.

Validation of the simulation with extreme precision requires the exact stress, or strain, values from the experimental work. This is done, according to Fitzpatrick et al. [30], using Bragg's law and the scattering of either X-rays or neutrons. To obtain the spacing, the part is placed in the apparatus and the diffraction patterns are recorded from various angles. This allows for a baseline pattern set which gives the starting spacing for all the atoms. The part is then put through the thermal process being investigated which 

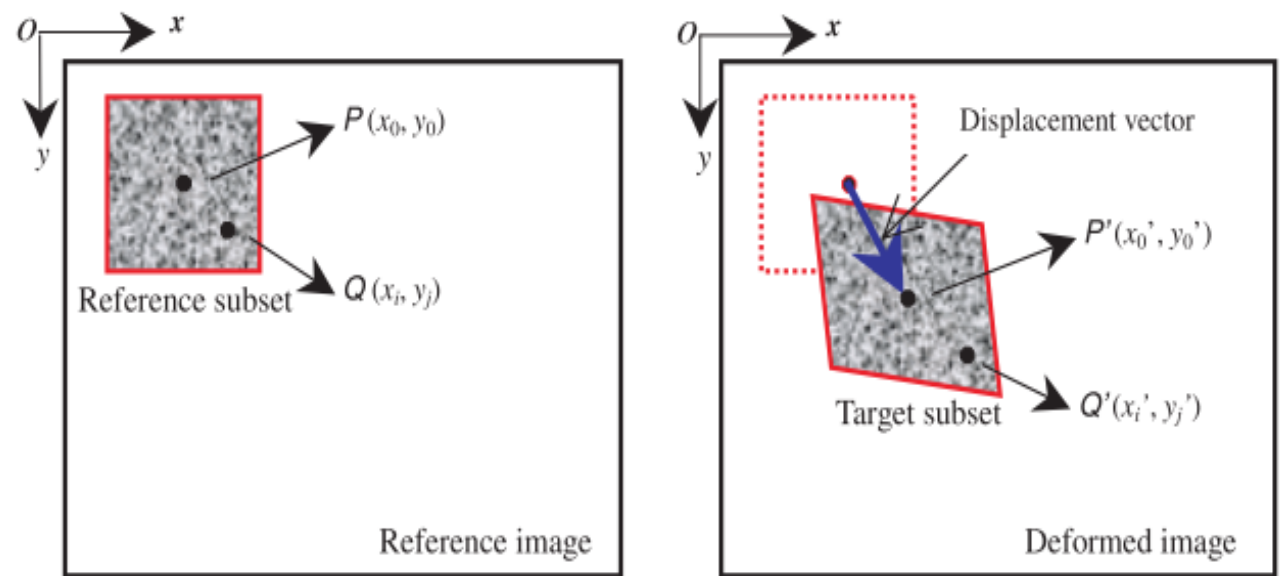

Fig. 4 Schematic showing displacement of tracking points in DIC [29].

will move the atoms. The difference in the diffraction patterns directly correlates to the distance that the atoms shifted. This motion of atoms is known as the strain which can then be converted to stress using Hooke's law.

This method of determining the stress locally allows for a direct correlation between the experiment and simulation. The choice of neutron or X-ray is based mainly on availability to the researchers. The use of XRD (X-ray diffraction) is much more widely available to researchers and therefore generally a more cost-effective method, whereas the use of neutrons is only done in specific facilities. One of the downfalls of these strain measurements is their inability to be used in-situ. Therefore the measurements are only of the final stresses. In addition to the localized strain, Ding et al. [16] have used the aforementioned 3-D scanners to further verify the simulations results.

\section{Microstructure Validation Techniques}

Due to its many desirable characteristics, namely its high strength to weight ratio and corrosion resistance, Ti-64 has been the focus of many researchers and leaders in industry. Because of this previous body of knowledge, this section will focus on Ti-64. However, these techniques can be applied to most metals. In many metals, and in particular Ti-64, the microstructure is critical to obtain optimal strength. Because of this, many researchers have developed models to determine the microstructure of an AM build.

To understand the modeling of the microstructure of Ti-64, it is necessary to study the microstructures that can occur. Ti-64, according to Kelly [31], has a microstructure which is a combination of a BCC (body-centered cubic), which is denoted as a $\beta$ phase, and an HCP (hexagonally closed packet), which is denoted as an $\alpha$ phase. These phases will coexist within the Ti-64 part and the quantities and sizes will depend on the maximum temperature and cooling rate at a specific location. At room temperature, the typical micro-structure is $\alpha+\beta$. If the material's temperature is raised higher than the beta transus temperature the material will transition into pure beta phase. As the material cools, the alpha phase will reappear and the cooling rate will dictate which alpha phases occur. This is shown graphically in Fig. 5. If the cooling rate is fast then the resulting alpha phase will be Martensitic $\left(\alpha_{0}\right)$ or Massive $\left(\alpha_{m}\right)$. These phases will appear intra-granularly and on the grain boundaries respectively. On the contrary, if the cooling rate is slow then the resulting micro-structure will start with Allotriomorphic $\left(\alpha_{\mathrm{GB}}\right)$ on the grain boundaries followed by primary-alpha $\left(\alpha_{\mathrm{P}}\right)$, which is simply any alpha phase that appears from cooling above the beta transus temperature, which is shown in the BSE (back-scattered electron) graph in Fig. 6. Lastly, when the material containing $\alpha_{P}+\beta$ is heated, but not past the 
beta transus temperature, some of the $\alpha_{P}$ will convert to $\beta$. When this material then cools, the new phase created is called secondary-alpha $\left(\alpha_{S}\right)$. This secondary phase becomes critical in AM due to the constant reheating from the layer by layer manufacturing strategy. Based on this understanding of the micro-structure evolution there are a few methods of quantifying, and therefore validating, a simulation which are outlined in Table 5.

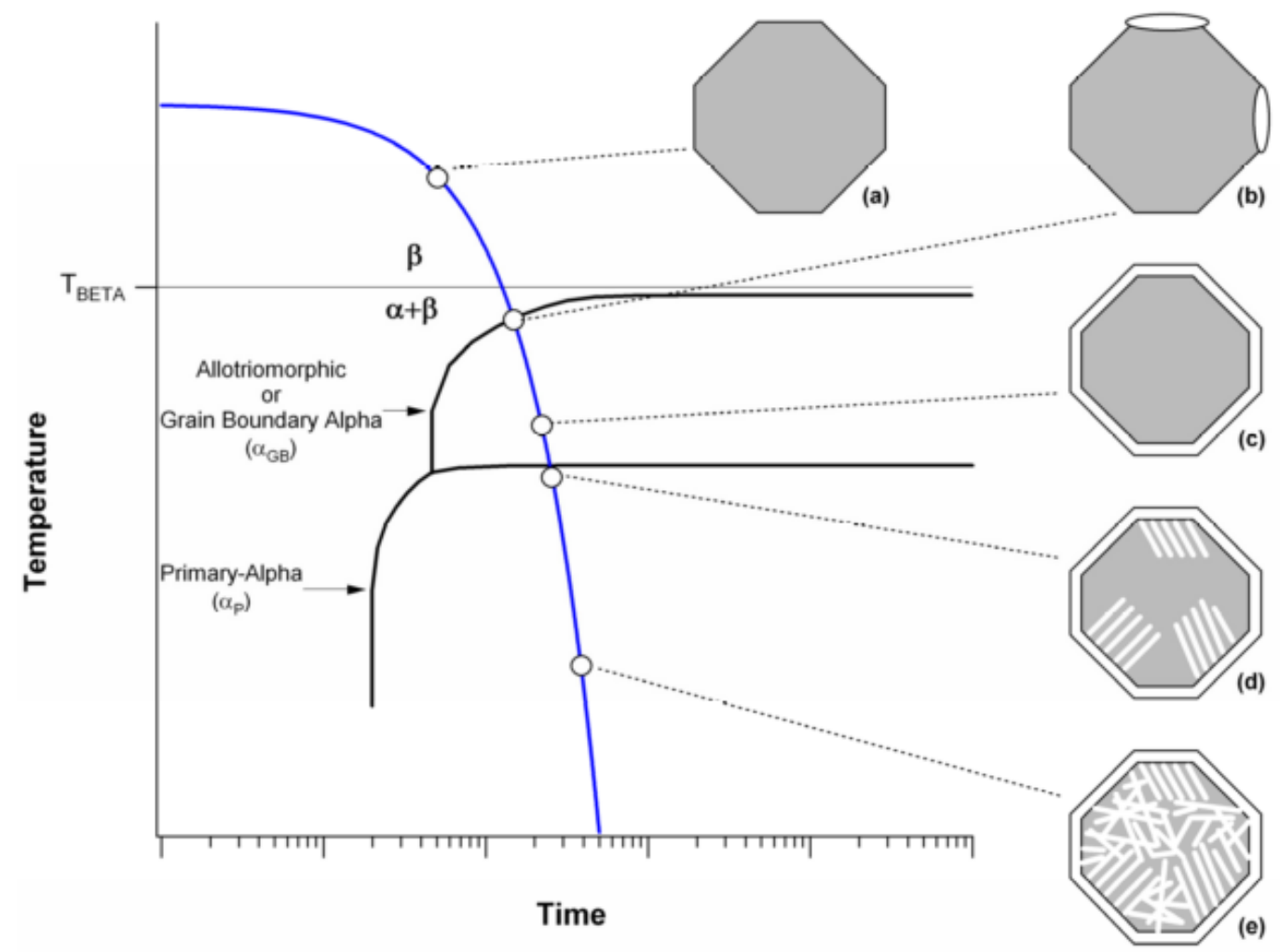

Fig. 5 Phase transformations which occur in Ti-64 [31].

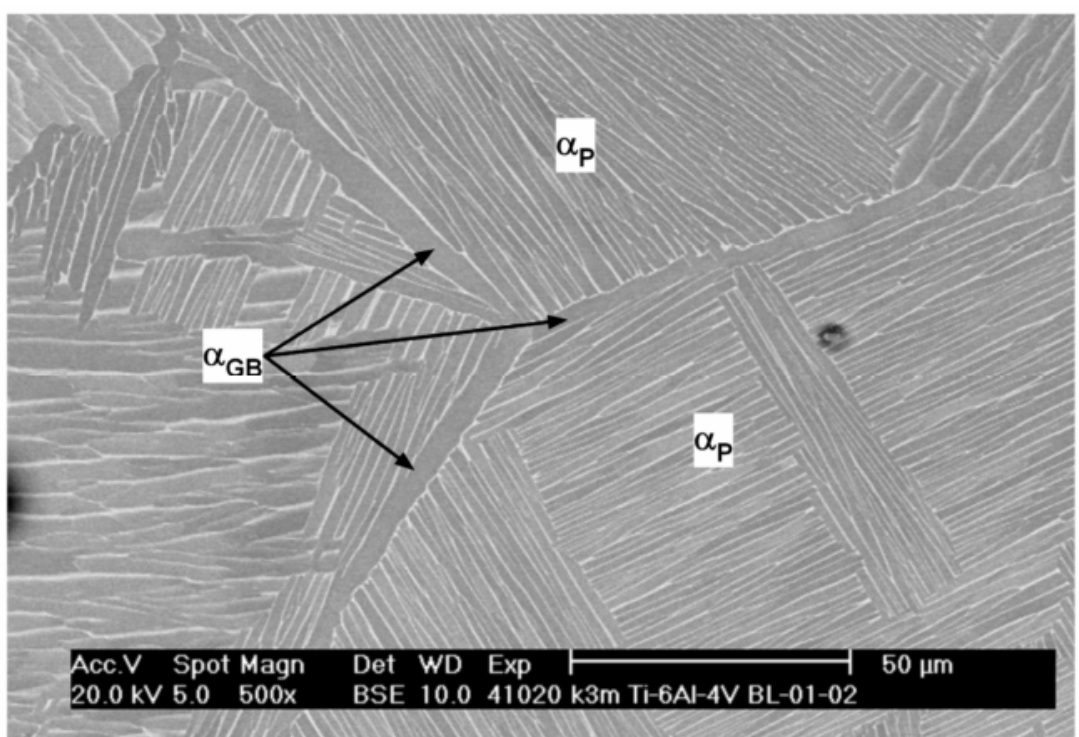

Fig. 6 Phases of Ti-64 [31]. 
Table 5 Frequency of micro-structure analysis techniques.

\begin{tabular}{ll}
\hline Element Wise Comparison & {$[32]$} \\
Phase Volume Comparison & {$[33,34]$} \\
Grain Size Distribution & {$[9,33,35]$} \\
\hline
\end{tabular}

In the first simulation method, by Kelly et al. [32], the elements are only allowed to be one of the various phases. Based on the elements thermal history, it is denoted as either beta or one of the alpha phases. This allows for a very general comparison with experimental results. When a thin wall is built, it can be sliced perpendicular to the laser scanning direction. This slice can then be observed with the SEM (scanning electron microscope). These images will then produce distinct regions, as shown in Fig. 7, of each phase which can be compared to simulations.

This simplified method is a fundamental start but is very lacking. Metallurgy has shown that the grain size, morphology, and distribution of fine particles are just as important to the mechanical properties as the phase itself. Therefore, Murgau et al. [34] have attempted to model the grain size along with the phase. The simplest of these validations use the volume percent of each of the phases. To ensure that their solution is robust, several cooling rates were modeled and compared to experimental results. When several cooling rates simulated matched experimental results, the simulation was considered correct, which is illustrated in Fig. 8.
Another method of validating the micro-structure is by comparing the size distribution of the alpha phase, which was done by Charles [35]. To compare the size distribution of the alpha phase, the average width of the alpha phases can be calculated and this can be used to compare the simulation to the experimental data. In order to be more rigorous Katzarov et al. [33] created a histogram of the sizes of the alpha phase in addition to the use of the volume percent of the phases. All in all, if a more detailed and rigorous validation technique is used the simulation can be more trusted.

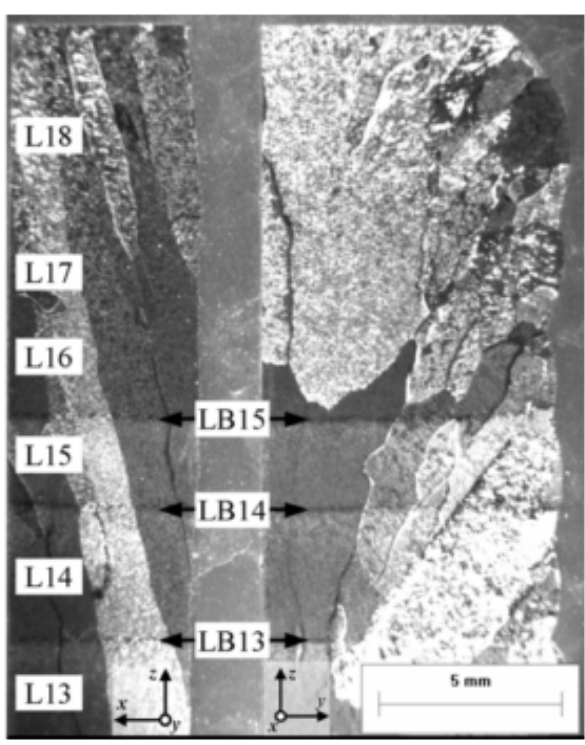

Fig. 7 Phase layers of Ti-64 produced via thin wall deposition [32].

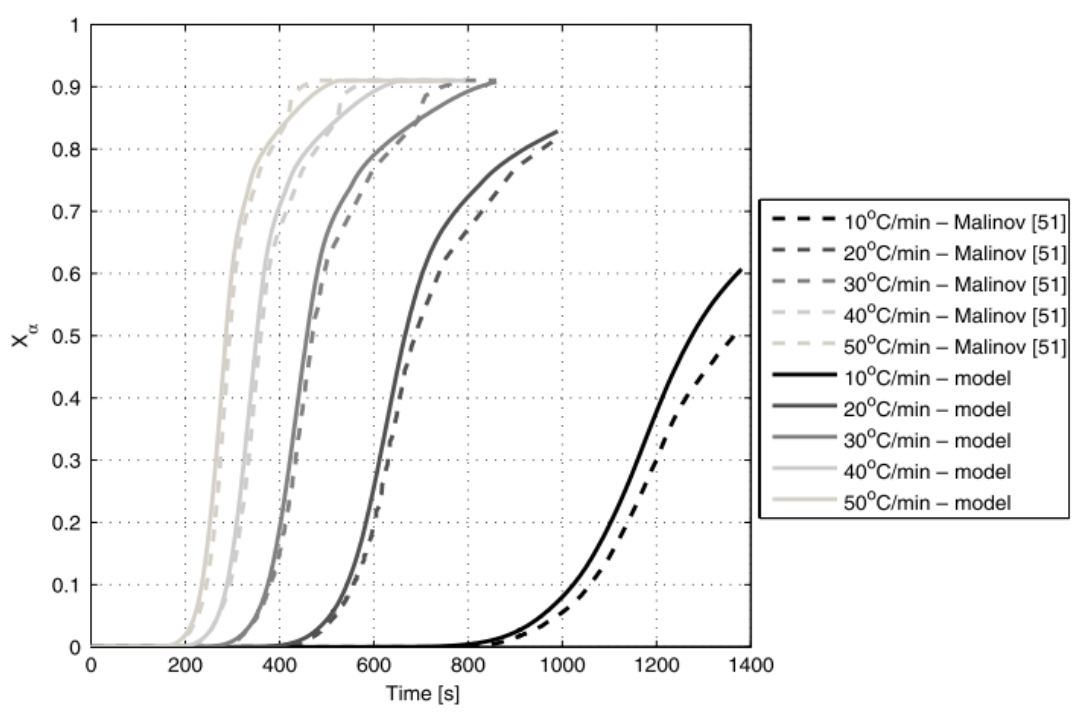

Fig. 8 Volume fraction of alpha phase comparison [34]. 


\section{Conclusions}

This paper presents the main validation techniques in literature for the validation of thermal modeling of metal AM and other attributes which are related to the thermal history. The heat transfer in the build can be measured using either direct or indirect means. The direct means include the use of cameras, pyrometers, and thermocouples. These methods give a direct link between the mathematical models and the experimental data. The indirect methods of validation use the melted track dimensions to show that the simulation is correct. This method relies heavily on the fluid model being correct as well as the correctness of the thermal model. Because of this, it can be preferred to use a direct method of measuring the heat flow.

Closely linked to the thermal history are the stresses induced in the build. To verify the modeling of stresses developed during a build, some have used the presence of cracks. This is only a rough correlation and to be more precise the parts distortion, during and after the build, can be analyzed, along with distortions which occur after selective sectioning to reveal the induced stresses, lastly to directly measure the strain diffraction that needs to be utilized to measure the shift of the atoms within the material.

In addition to the stress, the microstructure of Ti-64 is mainly dependent on the thermal history. The validation of this simulation can take a crude form of validation based solely on the phase present. A more rigorous approach involves calculating the percent volume of each of the phases and comparing these values. In addition, the size distribution of a phase can be found which can be used for more robust validation. All in all, the validation of simulation is very critical and sometimes an overlooked step. The selection of a validation technique must be appropriate for the simulation which is being created.

\section{References}

[1] Mackwood, A., and Crafer, R. 2005. “Thermal Modelling of Laser Welding and Related Processes: A Literature
Review.” Optics \& Laser Technology 37: 99-115.

[2] Mazumder, J., and Steen, W. 1980. "Heat Transfer Model for CW Laser Material Processing.” Journal of Applied Physics 51: 941-7.

[3] Chande, T., and Mazumder, J. 1981. "Heat Flow during CW Laser Materials Processing.” In Lasers In Metallurgy: Proceedings of a Symposium, TMS-AIME, 165-77.

[4] Reed, R., and Bhadeshia, H. 1994. "A Simple-Model for Multi-pass Steel Welds.” Acta Metall Mater 42: 3663-78.

[5] Lindgren, L., Runnemalm, H., and Nasstrom, M. 1999. "Simulation of Multi-pass Welding of a Thick Plate." International Journal For Numerical Methods In Engineering 44: 1301-16.

[6] Scott, D. A., and Frewin, M. R. 1999. "Finite Element Model of Pulsed Laser Welding.” Weld. J. 78 (1): 15-22.

[7] Hu, D., and Kovacevic, R. 2003. "Sensing, Modeling and Control for Laser-Based Additive Manufacturing." International Journal of Machine Tools and Manufacture 43: 51-60.

[8] Kolossov, S., Boillat, E., Glardon, R., Fischer, P., and Locher, M. 2004. "3D FE Simulation for Temperature Evolution in the Selective Laser Sintering Process." International Journal of Machine Tools and Manufacture 44: 117-23.

[9] Zhu, G., Zhang, A., Li, D., Tang, Y., Tong, Z., and Lu, Q. 2011. "Numerical Simulation of Thermal Behavior during Laser Direct Metal Deposition.” The International Journal of Advanced Manufacturing Technology 55 (9-12): 945-54.

[10] Zeng, K., Pal, D., and Stucker, B. E. 2012. "A Review of Thermal Analysis Methods in Laser Sintering and Selective Laser Melting.” In Proceedings of the Solid Freeform Fabrication Symposium, 796-814.

[11] Contuzzi, N., Campanelli, S. L., and Ludovico, A. D. 2011. "3D Finite Element Analysis in the Selective Laser Melting Process." International Journal of Simulation Modelling 10 (3): 113-21.

[12] Khairallah, S. A., and Anderson, A. 2014. "Mesoscopic Simulation Model of Selective Laser Melting of Stainless Steel Powder." Journal of Materials Processing Technology 214 (11): 2627-36.

[13] Tang, Q., Pang, S., Chen, B., Suo, H., and Zhou, J. 2014. "A Three Dimensional Transient Model for Heat Transfer and Fluid Flow of Weld Pool during Electron Beam Freeform Fabrication of Ti-6-Al-4-V Alloy.” International Journal of Heat and Mass Transfer 78: 203-15.

[14] Dai, K., Li, X., and Shaw, L. 2004. "Comparisons between Thermal Modeling and Experiments: Effects of Substrate Preheating.” Rapid Prototyping Journal 10 (1): 24-34. 
[15] Peyre, P., Aubry, P., Fabbro, R., Neveu, R., and Longuet, A. 2008. "Analytical and Numerical Modelling of the Direct Metal Deposition Laser Process.” Journal of Physics D: Applied Physics 41 (2): 025403.

[16] Ding, J., Colegrove, P., Mehnen, J., Ganguly, S., Almeida, P. M. S., Wang, F., and Williams, S. 2011. "Thermo-mechanical Analysis of Wire and Arc Additive Layer Manufacturing Process on Large Multi-layer Parts.” Computational Materials Science 50 (12): 3315-22.

[17] Heigel, J. C., Michaleris, P., and Reutzel, E. W. 2015. "Thermo-mechanical Model Development and Validation of Directed Energy Deposition Additive Manufacturing of Ti-6Al-4V.” Additive Manufacturing 5: 9-19.

[18] Wegner, A., and Witt, G. 2011. "Process Monitoring in Laser Sintering Using Thermal Imaging.” In Proceedings of the Solid Freeform Fabrication Symposium, 405-14.

[19] Fischer, P., Locher, M., Romano, V., Weber, H. P., Kolossov, S., and Glardon, R. 2004. "Temperature Measurements during Selective Laser Sintering of Titanium Powder." International Journal of Machine Tools and Manufacture 44 (12-13): 1293-6.

[20] Zhang, Y. 1998. "Thermal Modeling of Advanced Manufacturing Technologies: Grinding, Laser Drilling, and Solid Freeform Fabrication.” PhD thesis, University of Connecticut.

[21] Ding, J., Colegrove, P., Mehnen, J., Williams, S., Wang, F., and Almeida, P. S. 2014. “A Computationally Efficient Finite Element Model of Wire and Arc Additive Manufacture." International Journal of Advanced Manufacturing Technology 70 (1-4): 227-36.

[22] Liu, H., Sparks, T. E., and Liou, F. W. 2013. "Numerical Analysis of Thermal Stress and Deformation in Multi Layer Laser Metal Deposition Process.” In Proceedings of the Solid Freeform Fabrication Symposium, 577-91.

[23] Gusarov, A. V., Pavlov, M., and Smurov, I. 2011. "Residual Stresses at Laser Surface Remelting and Additive Manufacturing.” Physics Procedia 12: 248-54.

[24] Denlinger, E. R., Irwin, J., and Michaleris, P. 2014. "Thermo-mechanical Modeling of Additive Manufacturing Large Parts.” Journal of Manufacturing Science and Engineering 136 (6): 061007.

[25] Wu, A. S., Brown, D. W., Kumar, M., Gallegos, G. F., and King, W. E. 2014. “An Experimental Investigation into Additive Manufacturing-Induced Residual Stresses in 316
L Stainless Steel.” Metallurgical and Materials Transactions A: Physical Metallurgy and Materials Science 45 (13): 6260-70.

[26] King, W., Anderson, A. T., Ferencz, R. M., Hodge, N. E., Kamath, C., and Khairallah, S. A. 2015. "Overview of Modelling and Simulation of Metal Powder Bed Fusion Process at Lawrence Livermore National Laboratory.” Materials Science and Technology 31 (8): 957-68.

[27] Yadroitsava, I., and Yadroitsev, I. 2013. "Residual Stress in Metal Specimens Produced by Direct Metal Laser Sintering." In Proceedings of the Solid Freeform Fabrication Symposium, 1689-99.

[28] Shah, K., Izhar, U. H., Shah, S. A., Khan, F. U., Khan, M. T., and Khan, S. 2014. "Experimental Study of Direct Laser Deposition of Ti-6Al-4V and Inconel 718 by Using Pulsed Parameters." The Scientific World Journal 2014: 841549.

[29] Pan, B., Qian, K., Xie, H., and Asundi, A. 2009. "Two-Dimensional Digital Image Correlation for in-Plane Displacement and Strain Measurement: A Review." Measurement Science and Technology 20 (6).

[30] Fitzpatrick, M. E., Fry, A. T., Holdway, P., Kandil, F. A., Shackleton, J., and Suominen, L. 2005. "Determination of Residual Stresses by X-ray Diffraction-Issue 2.” Measurement Good Practice Guide (52): 74.

[31] Kelly, S. M. 2004. "Thermal and Microstructure Modeling of Metal Deposition Processes with Application to Ti-6Al-4V.” PhD thesis, Virginia Polytechnic Institute and State University.

[32] Kelly, S. M., Babu, S. S., David, S. A., Zacharia, T., and Kampe, S. L. 2004. "A Thermal and Microstructure Model for Laser Deposition of Ti-6Al-4V.” 45-52.

[33] Katzarov, I., Malinov, S., and Sha, W. 2002. "Finite Element Modeling of the Morphology of $\beta$ to $\alpha$ Phase Transformation in Ti-6Al-4V Alloy.” Metallurgical and Materials Transactions A 33A: 1027-40.

[34] Murgau, C. C., Pederson, R., and Lindgren, L. E. 2012. “A Model for Ti-6Al-4V Microstructure Evolution for Arbitrary Temperature Changes." Modeling and Simulation in Materials Science and Engineering 20: 055006.

[35] Charles, C. 2008. "Modelling Microstructure Evolution of Weld Deposited Ti-6Al-4V.” PhD thesis, Lulea University of Technology. 